\title{
ILCEA
}

Revue de l'Institut des langues et cultures

d'Europe, Amérique, Afrique, Asie et Australie

$32 \mid 2018$

Didactique des langues et cultures de spécialité : méthodes, corpus et nouvelles technologies

\section{Compilation de normes de traduction par l'annotation de corpus parallèles bilingues}

Compilation of Translation Standards by the Annotation of Parallel Bilingual Corpus

Éric Poirier

\section{OpenEdition}

Journals

Édition électronique

URL : http://journals.openedition.org/ilcea/4839

DOI : 10.4000/ilcea.4839

ISSN : 2101-0609

Éditeur

UGA Éditions/Université Grenoble Alpes

Édition imprimée

ISBN : 978-2-37747-059-4

ISSN : $1639-6073$

Référence électronique

Éric Poirier, «Compilation de normes de traduction par l'annotation de corpus parallèles bilingues », ILCEA [En ligne], 32 | 2018, mis en ligne le 01 juillet 2018, consulté le 01 mai 2019. URL : http:// journals.openedition.org/ilcea/4839 ; DOI : 10.4000/ilcea.4839

Ce document a été généré automatiquement le 1 mai 2019.

(c) ILCEA 


\title{
Compilation de normes de traduction par l'annotation de corpus parallèles bilingues
}

\author{
Compilation of Translation Standards by the Annotation of Parallel Bilingual \\ Corpus
}

Éric Poirier

\section{Introduction}

1 Le présent article rend compte d'un travail empirique sur la compilation des normes de traduction mises au jour par la corrélation de trois catégories d'annotations (sémantique, syntaxique et traductologique) des segments d'un corpus de 91 occurrences spécialisées de business choisies aléatoirement dans le concordancier bilingue Tradooit (2017). Ce travail vise à contribuer à l'étude de la traduction des langues de spécialité au moyen de corpus bilingues parallèles de traduction. Il a consisté à dépouiller des occurrences dans un corpus parallèle anglais-français pour établir des normes de traduction textuelles à partir des acceptions spécialisées des termes en langue source et de leur traduction, au moyen de traits sémantiques et de patrons lexicogrammaticaux corrélatifs aux occurrences prélevées dans les textes sources. Les annotations sémantiques et syntaxiques des segments sources, à savoir la typologie des acceptions et des patrons lexicogrammaticaux, est tirée en bonne partie des méthodes décrites dans Poirier (2015) et Poirier (2016a) pour les besoins de l'exploitation de corpus unilingues en langue source dans l'interprétation des occurrences de business.

2 Notre choix s'est arrêté sur le concept business parce qu'il s'agit d'un concept nominal dénotatif (non prédicatif) qui entre dans la composition de nombreux termes et expressions spécialisés, monolexémiques ou multilexémiques. Cette fréquence d'emploi donne à penser que les modalités de traduction de business occupent une part appréciable 
des compétences à acquérir dans la traduction spécialisée, notamment en économie et en finance.

3 Pour les besoins du présent article, les occurrences du corpus prélevées aléatoirement dans le concordancier bilingue sont des occurrences qui appartiennent à la langue spécialisée. Cela signifie que les occurrences du concept dont les acceptions relèvent de la langue générale ont été exclues du corpus ${ }^{1}$. L'élément business compte en effet plusieurs acceptions de la langue générale, et le partage entre celles-ci et celles qui relèvent de la langue de spécialité avait déjà été établi dans les travaux précédents. En outre, nous posons que la direction de l'opération de traduction de la langue source à la langue cible dans les occurrences relevées est celle de la traduction de l'anglais au français.

4 L'analyse des corrélations textuelles des occurrences de business et de leur traduction revêt deux intérêts principaux. Le premier concerne la traductologie, et plus précisément la traductologie descriptive. De la même façon que les textes unilingues peuvent contribuer à l'étude de l'utilisation des éléments du lexique (Hanks, 2013), les corpus parallèles peuvent contribuer à l'étude des choix de traduction rationnels effectués dans un contexte professionnel et donner lieu à des généralisations fondées sur les usages. La méthode de la traductologie descriptive que nous décrivons vise à transformer des ressources primaires ou des données brutes présentes dans les corpus parallèles, lesquels fournissent des solutions de traduction professionnelle, en ressources secondaires, lesquelles fournissent des méthodes, des consignes et des normes prescriptives de traduction. Autrement dit, le travail que nous proposons vise dans un premier temps à transformer ce que Toury $(2012: 87)$ appelle les normes textuelles en normes extratextuelles, lesquelles représentent un discours théorique prescriptif sur la traduction qui se fonde sur les normes descriptives extraites des ressources textuelles.

En deuxième lieu, l'utilisation des corpus parallèles est utile pour la constitution d'une matière riche en vue de l'enseignement de la traduction professionnelle. Les formateurs peuvent ainsi découvrir et compiler des normes de traduction des concepts clés et les expliquer aux étudiants pour qu'ils les appliquent dans la traduction des textes pour lesquels ils sont évalués. Cette fonction pédagogique des corpus parallèles permet d'illustrer les techniques de traduction par des exemples concrets, enracinés dans l'emploi des concepts dans les textes, et à montrer aux étudiants comment appliquer les techniques des professionnels. La compilation de normes textuelles de traduction contribue à la modélisation des méthodes de travail des traducteurs professionnels et aux travaux de recherche sur les procédés de traduction (lorsque les solutions de traduction sont associées à des tâches cognitives précises) et sur l'évaluation de la qualité des traductions en traductologie. Comme les étudiants consultent déjà abondamment les corpus parallèles lorsqu'ils travaillent aux traductions qu'on leur demande de produire dans leur formation, la méthode que nous proposons, et qui consiste à découvrir et à compiler des normes textuelles de traduction professionnelle à partir de corpus parallèles, remplit aussi une fonction théorique utile pour le domaine et les étudiants de niveaux plus avancés qui peuvent apprendre à mieux exploiter les données brutes auxquelles ils ont accès pour être à même de constater et d'appliquer de nouvelles normes textuelles de traduction professionnelle. 


\section{Définitions}

6 Nous définissons ci-après quelques concepts qui jouent un rôle clé dans le travail d'analyse qui a été mené dans le dépouillement des normes textuelles de traduction des occurrences de business. Nous commençons par définir ce qui est entendu par normes de traduction dans le cadre théorique de la traductologie de corpus. Nous définissons ensuite brièvement ce qui est entendu par unité phraséologique et collocation, et leurs liens avec les notions de termes simples et complexes.

\subsection{Normes de traduction}

7 La compilation des normes textuelles de traduction des occurrences de business s'appuie tout d'abord sur le concept de ce que Toury (2012 : 79) appelle la « norme initiale » et qu'il définit comme un continuum ou un compromis à faire entre le pôle de l'adéquation de la traduction (dans son degré de similitudes statique et fonctionnelle avec le texte source) et son acceptabilité (dans son degré de respect des normes linguistiques, textuelles et culturelles de la langue-culture cible). Puisque les traductions des occurrences de business qui seront examinées proviennent d'un corpus parallèle de textes traduits, il semble acquis que les traductions relevées dans la compilation des normes seront acceptables au sens ci-dessus puisqu'elles ont été publiées en l'état ${ }^{2}$. Le travail de compilation des normes textuelles de traduction portera donc principalement sur le pôle de leur adéquation par rapport au texte source. Deux grandes mesures de similitude seront d'abord évaluées, à savoir leur nature directe ou indirecte. Ces mesures de similitudes définissent en quelque sorte deux grands procédés de traduction qui comportent chacun différentes techniques de traduction.

8 Les procédés de traduction directs désignent des correspondances, conventionnelles ou non, qui sont fondées sur le sens et sur la forme d'un concept, d'une notion ou d'une unité lexicale du texte source avec un autre concept, une autre notion, une unité lexicale du texte cible, dont la présence manifeste ou exprime une stratégie de traduction littérale ou mot à mot. Parmi les solutions directes, nous avons distingué les traductions conventionnelles des traductions non conventionnelles. Les premières correspondent aux traductions que l'on retrouve dans la plupart des ouvrages de traduction spécialisés, et leurs synonymes immédiats tels que ceux que l'on trouve dans le Dictionnaire électronique des synonymes (DES) (1998-2018). Les deuxièmes désignent toutes les traductions mot à mot qui ne sont pas des traductions conventionnelles et qui ne sont pas nécessairement littérales. Nous avons appelé les traductions non conventionnelles les ruptures sémantiques du fait qu'elles respectent la construction syntaxique du texte source, mais que leur acception s'écarte considérablement des sentiers battus ou des traductions habituellement utilisées dans le microcontexte. Le concept de rupture est l'équivalent en français de ce qu'on appelle un translation shift en anglais défini notamment par Bakker, Koster et Van Leuven-Zwart (2011).

9 Le deuxième groupe de procédés de traduction indirects réunit les solutions de traduction qui ne procèdent pas du principe de traduction précédent qui consiste à rendre chacune des unités lexicales du texte source par une unité lexicale de la langue cible, que ce soit dans son sens littéral ou par rupture sémantique. Définies de manière positive, les solutions de traduction indirectes sont caractérisées par une rupture 
formelle, laquelle peut être accompagnée ou non d'une rupture sémantique. Nous avons réparti les solutions de traduction indirectes entre les ruptures formelles (dont le sens littéral est assimilable au sens ou à l'acception de business dans le texte source), les ruptures formelles et sémantiques qui expriment une rupture à la fois formelle (traduction qui n'est pas mot à mot) et sémantique (traduction qui n'est pas assimilable à une traduction littérale) ainsi que, ce que nous avons appelé, les tradaptations inanalysables dont la relation avec le texte source est difficile à établir autrement que sur le plan fonctionnel (elles figurent dans le même microcontexte textuel que business dans le texte source, mais elles n'entretiennent aucun rapport conventionnel (lexical ou sémantique) avec l'occurrence de business).

10 La présente étude sur la traduction de business s'inspire en partie de travaux sur les normes d'exploitation des éléments du lexique de Hanks (2013) dans le domaine unilingue et sur l'approche lexicogrammaticale de Gledhill (2015) dans l'étude des solutions de traduction de qualité en situation de révision ou de traduction. Ces travaux ainsi que ceux qui adoptent une perspective similaire portent presque exclusivement sur l'utilisation ou la traduction d'occurrences verbales, et accordent de ce fait peu d'attention aux occurrences nominales, lesquelles sont pourtant plus nombreuses en langue de spécialité, comme l'atteste le contenu presque exclusivement nominal de n'importe quelle banque de terminologie, ou des vocabulaires et lexiques spécialisés.

11 Comme l'explique Toury (2012: 87), les normes de traduction ne sont pas observables en tant que telles, et ce sont plutôt les comportements normalisés ou leurs produits qui peuvent être observés à partir de sources textuelles ou extratextuelles. Dans notre étude, nous nous intéressons uniquement aux sources textuelles des normes de traduction qui désignent ici des données linguistiques pour les annotations sémantiques et syntaxiques, et des faits de traduction pour les annotations traductologiques. Ces données ont été recueillies par l'analyse des annotations et de leurs corrélations entre elles. Les techniques de traduction décrivent les solutions de traduction réellement utilisées dans les occurrences attestées dans le corpus parallèle de traductions. Par exemple, la traduction de business par société en français constitue une méthode de traduction directe d'une acception particulière de business valable dans l'un de ses nombreux emplois (syntaxiques) qui doivent être précisés dans la description de la méthode de traduction. La méthode est censée fournir de l'information sur la façon de traduire une acception de business ainsi que sur les conditions qui déterminent la façon de la traduire.

\subsection{Unités phraséologiques et collocations}

12 Notre étude vise aussi bien les occurrences simples (unitaires ou monolexémiques) de business que celles qui participent à des termes et expressions complexes (multilexémiques). Dans le domaine des langues de spécialité, les termes complexes particulièrement fréquents et conventionnels (dont le sens est stable) et les expressions font partie de ce que l'on appelle plus généralement les unités phraséologiques. Ces unités linguistiques particulières, qui font l'objet de la phraséologie, réunissent un ensemble très varié d'unités composé de locutions, composés, proverbes, formules, citations connues, etc. Du fait de leur nature variable, elles sont difficiles à définir. Une définition consensuelle du phénomène, comme celle de Gries (2008:6), met en évidence le fait que les unités phraséologiques comprennent deux unités lexicales qui ont un sens particulier et dont la fréquence de cooccurrence est plus forte qu'une distribution 
aléatoire ${ }^{3}$. Ce sont ces caractéristiques qui nous autorisent à réunir dans ce groupe, au moins pour les besoins de la présente étude de corpus, les termes complexes qui appartiennent bien entendu aux langues de spécialité, mais aussi les autres composés et expressions formés avec une occurrence de business dans l'une de ses acceptions spécialisées.

13 Selon que la forme obtenue donne lieu à un terme ou à une expression complexe (non terminologisée), la contribution de business à une expression complexe relève de la néologie terminologique ou de la combinatoire syntaxique. Le statut terminologique et sémantique de l'élément constitutif demeure ambigu puisqu'il est parfois dissous dans l'ensemble dont il fait partie, ou alors il reste un terme à part entière doté d'un sens propre. Les déterminismes qui sont à l'œuvre dépendent à la fois du degré de terminologisation de l'expression, qui n'est pas facile à mesurer, à l'instar de la définition concrète et pratique du néologisme qui constitue, selon Humbley (2002:92), un grave problème, mais aussi du statut de la collocation comme phénomène régulier ou ordinaire dans les études phraséologiques qui portent plus spécialement sur les expressions qui relèvent d'une interprétation combinatoire irrégulière ou extraordinaire (Tutin \& Grossman, 2002). La terminologie de la néologie est ainsi dépourvue d'une nuance qui serait fort utile entre le terme simple, unitaire, et le même terme entrant dans la composition d'un terme complexe. Ces deux emplois d'un même terme représentent-ils le même type d'unité terminologique ? Plutôt que de trancher sur cette question ou même de l'aborder d'un point de vue déterministe, nous avons utilisé chaque fois que cela nous est apparu possible un terme différent. Le générique concept sert à indiquer que le sens de business conserve son autonomie relative dans l'expression, tandis que le générique élément sert à indiquer qu'il y est dissout. Lorsqu'il n'était pas pertinent d'établir la distinction entre les deux utilisations de business, nous avons utilisé l'expression «occurrences de business ».

\section{Objectifs}

14 L'analyse du corpus d'occurrences de business vise à dégager des généralisations sur les modalités de traduction des acceptions spécialisées de business en français. La mise en corrélation des annotations sémantiques et syntaxiques avec les annotations traductologiques permettra d'établir une vue d'ensemble des procédés de traduction s'appliquant aux différentes acceptions et aux différents patrons syntaxiques de business. La vue d'ensemble permettra de décrire des règles conditionnelles et des normes variables de traduction utiles aux traducteurs professionnels. Nous espérons aussi que ces normes de traduction seront utiles aux enseignants dans la conception d'activités d'apprentissage pertinentes sur les emplois spécialisés de business et sur leurs méthodes de traduction. Une meilleure connaissance des principes généraux de traduction des occurrences de business, des fréquences d'emploi de business et de ses modalités de traduction favorisera la conception d'exercices de traduction à difficulté croissante tenant compte du niveau des étudiants et des emplois de business qui présentent des degrés de difficultés variables. 


\section{Méthodologie}

15 Nous avons commencé l'étude par la recherche d'un corpus parallèle fiable d'occurrences de business et de leur traduction. Notre choix s'est arrêté sur les résultats fournis par Tradooit (2017), un concordancier dont l'utilisation est gratuite et qui réunit un nombre élevé de documents alignés produits principalement au Canada, mais aussi en Europe. La provenance géographique des corpus qui constituent ce concordancier est un critère à ne pas négliger pour les besoins de la définition des normes de traduction au Québec et au Canada. Pour les besoins de la présente analyse, une recherche simple du concept business a été lancée en septembre 2016 dans Tradooit (2017) et les cent premiers résultats ont été conservés 4 .

L'analyse des occurrences de business repose sur trois grandes catégories d'annotations : les annotations sémantiques, les annotations syntaxiques et les annotations traductologiques. Nous décrivons ci-après les principes et les critères utilisés pour chaque type d'annotation dans un souci de description objective de la méthode utilisée et de sa reproductibilité pour d'autres éléments conceptuels des lexiques spécialisés ainsi que pour la compilation de normes de traduction de business vers d'autres langues.

\subsection{Les annotations sémantiques}

17 Les annotations sémantiques sont essentielles pour mieux rendre compte de la traduction qui porte sur la signification et sur le sens. Elles s'appuient sur la table d'interprétation décrite dans Poirier (2015) qui définit les huit acceptions spécialisées suivantes de business : 1a) «a professional occupation»; 1b) «administrative duties (especially of public organization such as parliament, government, committee or council)»; 2a) «series of commercial or economic activities»; $2 b$ ) «volume of commercial or economic activities »; 2c) « people or entity generating economic activities, i.e. customers »; $3 a)$ « a specific business concern, i.e. a company »; $3 \mathrm{~b}$ ) « all profit-making entities in society, i.e. the private sector »; $3 \mathrm{c}$ ) " a group of businesses having similar activities, i.e. an industry ». Après analyse de chacun des segments source, et indépendamment de leur traduction, une de ces huit acceptions a été attribuée à chaque occurrence de business. Quelques occurrences se sont avérées difficiles à analyser sur le plan de l'acception utilisée, notamment entre l'interprétation 2a ou 3a dans quelques contextes comme celui-ci :

Tableau 1. - Occurrences équivoques entre $2 a$ et $3 a$.

\begin{tabular}{l|l} 
Segment source & Segment cible \\
\hline Making product manufacturers and importers & Voir à ce que les fabricants et les importateurs \\
responsible for the recycling of their products and & recyclent leurs produits et leurs emballages - et leur \\
packaging - and giving them the flexibility to do so & $\begin{array}{l}\text { donner la possibilité de le faire d'une façon qui } \\
\text { in a way that best suits their business. }\end{array}$
\end{tabular}

18 L'acception consignée dans les annotations sémantiques est celle de la langue source et non pas l'acception "dérivée » que l'on peut obtenir par glissement de sens et par rupture sémantique dans la langue cible (voir la section 5.3). Le nombre d'acceptions et leur proportion relevée dans le corpus de 91 occurrences sont décrits à la section 5.2. 


\subsection{Les annotations syntaxiques}

19 Elles décrivent cinq patrons syntaxiques d'utilisation du nom business - mais en fait la typologie peut s'appliquer à tous les noms spécialisés - pour former des termes spécialisés monolexémiques et multilexémiques.

\subsubsection{Les UP}

Le premier patron qui a été annoté est celui de l'appartenance de business à une unité phraséologique (UP) reconnue comme telle dans les ouvrages lexicographiques et conforme à la définition de la section 2.2 ci-dessus. Il s'agit d'un groupe d'au moins deux unités lexicales se comportant comme un nom, un verbe ou un adverbe (voir les exemples plus loin) dans lequel l'acception de l'occurrence de business est non autonome ou indissociable de l'ensemble. Par définition, les UP n'actualisent aucune acception particulière de business qui fusionne sémantiquement avec l'acception de l'ensemble de l'expression, sauf pour ce qui est des collocations qui ne sont pas assimilées à des UP ici puisque leur sens est compositionnel. Contrairement à la traduction des autres patrons, la traduction des UP s'effectue globalement et ne s'appuie généralement pas sur une interprétation compositionnelle de l'acception de business dans le syntagme. Ce patron syntaxique doit être pris en compte le premier parce que l'interprétation phraséologique a toujours préséance sur l'interprétation libre des unités multilexémiques.

21 À titre d'exemples du premier patron syntaxique de business qui entre dans la formation d'unités phraséologiques, on trouve dans le corpus des expressions nominales comme line of business, agribusiness, verbales comme doing business as, do business with, to be in business with $s b$ et adverbiales comme for business, in business. On a aussi trouvé les expressions business-related et small business, mais leur statut phraséologique n'est pas clairement établi comme adjectif composé, de sorte que leur patron syntaxique a plutôt été annoté comme un modifieur complexe (voir plus loin).

\subsubsection{Les emplois unitaires}

Le deuxième patron annoté est celui des emplois unitaires du terme business. Dans ces occurrences, le critère retenu est le fait que le syntagme nominal dont l'élément business fait partie ne contient qu'un seul élément terminal, à savoir le concept business lui-même. C'est le cas, par exemple, de l'occurrence soulignée dans le segment parallèle du tableau 2 ci-dessous.

Tableau 2. - Occurrence unitaire du concept spécialisé de business.

Segment source
While the focus has traditionally been on direct
taxation, the customs duties dimension of transfer
pricing is now increasingly attracting the attention
of both governments and business.

Segment cible

Si l'accent était mis jusqu'à présent sur la fiscalité directe, les droits de douane liés aux prix de transfert suscitent de plus en plus l'attention des gouvernements et du secteur privé.

Même si business est coordonné avec governments, chacun des éléments forme un syntagme nominal à un seul élément terminal. L'exemple illustre ce qu'on appelle un binôme, ou binomial en anglais, lequel coordonne deux syntagmes nominaux ou éléments nominaux terminaux dans un même syntagme. La coordination fait intervenir une règle 
générale d'interprétation qu'il n'a pas semblé pertinent d'étudier ici puisqu'elle ne s'applique pas spécialement à business. La règle fait partie des procédés d'interprétation des noms qui sont coordonnés à des antonymes ou à des éléments complémentaires sur le plan notionnel. Ce type de règle facilite l'interprétation des éléments coordonnés en langue de spécialité et doit faire partie des principes généraux de traduction des noms ou concepts nominaux spécialisés. Dans le tableau 2, l'opposition sous-jacente à la coordination des deux noms est celle de deux secteurs économiques fondamentaux, à savoir le secteur public (le gouvernement) et le secteur privé (les entreprises).

\subsubsection{Les emplois syntagmatiques}

Les trois autres patrons annotés sont tous constitués de syntagmes nominaux dont les éléments terminaux comprennent l'élément business ainsi qu'au moins un ou plusieurs autres éléments lexicaux distincts de business qui lui sont étroitement liés parce que business tient le rôle de complément ou de modifieur (cette notion est définie ci-dessous).

Le troisième patron annoté est celui de business en tant que noyau du syntagme nominal, comme dans l'occurrence soulignée dans le segment parallèle du tableau 3 ci-dessous.

Tableau 3. - Occurrence de business comme noyau syntaxique.

\begin{tabular}{l|l} 
Segment source & \multicolumn{2}{l}{ Segment cible } \\
\hline $\begin{array}{l}\text { These would reportedly lead to further decay of the } \\
\text { railway business and deterioration in the quality of } \\
\text { services, and would force personnel to strike more, } \\
\text { for reasons of self-preservation. }\end{array}$ & $\begin{array}{l}\text { compagnies de chemin de fer et dégrader encore } \\
\text { plus leurs services, ce qui pousserait le personnel à } \\
\text { recourir à la grève comme moyen d'autodéfense. }\end{array}$
\end{tabular}

Le noyau est l'élément dans le syntagme qui détermine les accords grammaticaux (du sujet avec le verbe, par exemple) avec les autres constituants de la phrase. Il peut être intéressant de savoir si la fonction syntaxique de noyau n'est pas corrélée avec certaines acceptions et modalités de traduction particulières. Dans l'exemple cité, la traduction fournit aussi un exemple de rupture sémantique (voir plus loin) dans le passage entre l'acception $3 \mathrm{~b}$ et le pluriel de l'acception 3a qui ont une signification très semblable.

Lorsque business n'est pas le noyau du syntagme, ses occurrences ont été classées dans l'un ou l'autre de deux patrons syntagmatiques qui reposent sur la distinction entre les emplois de business comme modifieur simple, le quatrième patron syntagmatique de notre corpus, lequel modifie l'élément noyau du syntagme, et les autres rôles de business comme modifieur secondaire (modifieur de modifieur) d'un autre élément à l'intérieur du syntagme. Le terme modifieur désigne, dans un syntagme nominal, les éléments nominaux ou adjectivaux qui sont susceptibles de modifier le noyau du syntagme. Ce terme ne doit pas être confondu avec son paronyme, le terme modificateur, que l'office québécois de la langue française (2018) définit comme « la fonction syntaxique de l'adverbe ou du GAdv en relation avec un verbe, un adjectif ou un autre adverbe ».

Le cinquième patron des modifieurs complexes comprend différents rôles attribués à business, lorsqu'il fait partie, par exemple, avec le nom qu'il modifie, d'un noyau complexe, qui est lui-même modifié par un troisième élément dans le syntagme nominal, ou encore, lorsqu'il fait partie d'un syntagme modifieur qui modifie un autre concept noyau. On trouve ces cas de figure et d'autres configurations dans les occurrences suivantes: "small-sized business combinations ", "Business Corporation Act », « small business community », " business-related service enterprises », etc. 
29 Les rôles syntaxiques secondaires dans le patron syntaxique des modifieurs complexes n'ont pas été annotés en raison du petit nombre d'occurrences exemplifiant chacun d'eux. La prise en compte d'un nombre plus élevé d'occurrences contribuerait à l'établissement de normes de traduction pour ces différentes constructions de business utilisé comme modifieur secondaire. Pour les mêmes raisons, nous n'avons pas distingué dans les annotations les emplois attributifs et déterminatifs de business lorsque business est un modifieur simple. Les premiers sont des appositions, dont la fonction est comparable à celle d'un adjectif, et les deuxièmes sont des compléments du nom qu'ils modifient.

\subsection{Les annotations traductologiques}

Les annotations traductologiques caractérisent la nature de l'élément cible qui est aligné formellement, sémantiquement ou fonctionnellement à business et qui sert donc de traduction au concept. La première étape de l'annotation traductologique a consisté à trouver l'élément aligné à business et, le cas échéant, à en préciser la nature. Lorsqu'aucune unité lexicale ne peut être alignée à l'occurrence de business, celle-ci a été alignée à une occurrence vide. Pour mener à bien l'alignement de business, on a utilisé la méthode de l'alignement des mots pleins décrite dans Poirier (2016b). En résumé, les règles de cette méthode sont les suivantes: repérer les mots pleins d'un segment puis aligner chaque mot plein source à au moins un mot plein cible, ou à une occurrence vide, et inversement, aligner chaque mot plein du segment cible à au moins ${ }^{5}$ un mot plein source, ou à une occurrence vide. L'application de ces règles nécessite l'alignement de tous les mots ou de tout le contenu informatif des segments, ce qui augmente le temps consacré à ce type d'annotation.

31 Les annotations qui ont servi à caractériser les traductions de business ont été définies à la rubrique sur les normes de traduction. Elles comptent deux grands procédés de traduction, les traductions directes ou indirectes, que nous décrivons ci-après et qui se manifestent par cinq techniques de traduction type.

\subsubsection{Les traductions directes}

32 Les traductions directes comprennent la traduction conventionnelle (TC) par correspondance et la rupture sémantique (RS). Les TC sont des traductions en langue cible que l'on trouve le plus souvent dans les dictionnaires bilingues, ainsi que leurs synonymes, hyponymes, hyperonymes et métonymes. C'est le cas de la traduction directe « cantine » en français utilisée dans son acception hyponyme d'entreprise, ou encore de la traduction « traite » dans son acception hyponyme de commerce en français.

Les RS sont des traductions directes non conventionnelles qui, tout en utilisant un correspondant lexical, représentent néanmoins une rupture sémantique par rapport aux traductions conventionnelles de l'acception. La RS s'évalue par le fait que l'acception de la traduction en français ne correspond pas à l'acception de business en langue source. À titre d'exemple, on peut penser aux glissements d'acception qui se produisent à l'occasion par la traduction, et que nous décrivons à la section 5.3 avec les exemples du tableau 7. 


\subsubsection{Les traductions indirectes} autre concept en langue cible. Lorsqu'aucune correspondance n'était possible parce que le segment dans lequel business figure est inanalysable, on a utilisé le symbole IN. Les traductions indirectes de type IN sont au nombre de trois occurrences seulement.

\section{Analyse des annotations}

Comme la traduction porte essentiellement sur la restitution du sens d'un message ou d'un énoncé, une première préoccupation dans l'analyse des annotations a été de chercher à savoir quels sont les patrons syntaxiques que privilégie chacune des acceptions de business, un travail qui a été mené dans Poirier (2015) et en partie dans Poirier (2016a). Avant d'examiner les corrélations des acceptions et des patrons syntaxiques avec les modalités de traduction des occurrences de business, nous avons cherché à savoir si la distribution des acceptions relevées dans le corpus concorde avec la distribution des acceptions relevées dans le corpus établi dans Poirier (2015). Nous avons également brossé un portrait d'ensemble des procédés analysés pour les traductions de 
business dans le corpus. Comme les traductions directes comptent pour une bonne part des procédés de traduction utilisés, nous avons jugé utile d'examiner la fréquence et la répartition des correspondants pour chaque procédé de traduction.

D'autres variables extralinguistiques entrent en compte dans les procédés de traduction de business, comme le contexte de communication, le type de document dans lequel business est utilisé, la fonction et la formation de la personne qui l'emploie, les traductions antérieures de business dont les locuteurs et les traducteurs ont connaissance, etc. Ces éléments ne sont pas pris en compte dans la présente étude, non pas parce qu'ils ne sont pas pertinents, mais parce que les annotations utilisées n'ont pas permis de codifier et de déterminer avec certitude les valeurs de ces variables.

\subsection{Distribution des acceptions}

La répartition des acceptions spécialisées de business qui ont été relevées pour la totalité des 91 occurrences du corpus aléatoire est décrite dans le tableau 4 ci-dessous.

Tableau 4. - Acceptions spécialisées de business dans le corpus.

\begin{tabular}{|c|c|}
\hline Acception & Fréquence (\%) \\
\hline 1a) a professional occupation & $1(1 \%)$ \\
\hline $\begin{array}{l}\text { 1b) administrative duties (especially of public } \\
\text { organization such as parliament, } \\
\text { government, committee or council) }\end{array}$ & $10(11 \%)$ \\
\hline $\begin{array}{l}\text { 2a) series of commercial or economic } \\
\text { activities }\end{array}$ & $34(37 \%)$ \\
\hline $\begin{array}{l}\text { 2b) volume of commercial or economic } \\
\text { activities }\end{array}$ & $0(0 \%)$ \\
\hline $\begin{array}{l}\text { 2c) people or entity generating economic } \\
\text { activities, i.e. "customers" }\end{array}$ & $0(0 \%)$ \\
\hline $\begin{array}{l}\text { 3a) a specific business concern, i.e. "a } \\
\text { company" }\end{array}$ & $27(30 \%)$ \\
\hline $\begin{array}{l}\text { 3b) all profit-making entities in society, i.e. } \\
\text { "the private sector" }\end{array}$ & $15(16 \%)$ \\
\hline $\begin{array}{l}\text { 3c) a group of businesses having similar } \\
\text { activities, i.e. "an industry" }\end{array}$ & $4(4 \%)$ \\
\hline
\end{tabular}

Seulement cinq des huit acceptions spécialisées de business ont été relevées dans le corpus de 91 occurrences choisies aléatoirement. Cette situation coïncide avec le relevé des acceptions dans Poirier (2015) qui portait sur un autre corpus unilingue d'occurrences de business. Comme pour notre corpus, dont les occurrences sont toutes différentes, on n'a relevé aucune occurrence pour les acceptions $2 \mathrm{~b}$ et $2 \mathrm{c}$, ce qui donne à penser que ces deux acceptions représentent des emplois peu fréquents de business. Par ailleurs, les trois acceptions les plus fréquentes de notre corpus (2a, $3 a$ et $3 b$ ) sont les mêmes que celles qui ont été relevées dans le corpus précédent, même si elles se présentaient alors dans un ordre différent (3a suivie de $3 \mathrm{~b}$ puis de $2 \mathrm{a}$ ). À la différence du corpus précédent, on constate dans ce corpus-ci, un nombre plus élevé d'acceptions $1 b$. semble préférable de centrer la formation à la traduction de business sur les trois 
principales acceptions $2 \mathrm{a}, 3 \mathrm{a}$ et $3 \mathrm{~b}$ de business plutôt que sur ses autres acceptions spécialisées.

\subsection{Vues d'ensemble des procédés de traduction}

Le tableau 5 qui suit décrit le nombre d'occurrences de chaque procédé de traduction annoté défini à la section 4.3, ainsi que leur proportion sur la totalité des occurrences de business.

Tableau 5. - Fréquence absolue des procédés de traduction.

\begin{tabular}{cc|ccc}
\multicolumn{4}{c}{ Solutions directes } & \multicolumn{3}{c}{ Solutions indirectes } \\
\hline$T C$ & $\mathrm{RS}$ & $\mathrm{RFS}$ & $\mathrm{RF}$ & $\mathrm{IN}$ \\
$52(57,1 \%)$ & $12(13,2 \%)$ & $5(5,5 \%)$ & $19(20,9 \%)$ & $3(3,3 \%)$ \\
$64(70,3 \%)$ & & $27(29,7 \%)$ &
\end{tabular}

Le procédé le plus fréquent est la traduction conventionnelle (57,1\%) qui est suivie des ruptures formelles $(20,9 \%)$. Pour interpréter ces résultats correctement, il faudrait pouvoir les comparer aux procédés de traduction d'autres concepts spécialisés dont la fréquence est semblable dans le domaine de traduction économique et financière.

Les données sur les traductions indirectes montrent que près de $30 \%$ des solutions de traduction, soit une proportion assez importante, recourent à d'autres modes de traduction que la traduction littérale mot à mot. Si on évalue le degré de difficulté d'une unité lexicale par la probabilité de recourir à des solutions de traduction indirectes, il semble bien que la traduction de business présente une bonne proportion de celles-ci, et vraisemblablement un degré de difficulté de traduction conséquent, même si la valeur précise de la difficulté reste à déterminer au moyen d'analyses par corpus d'autres concepts spécialisés qui sont caractéristiques de la traduction économique, commerciale et financière.

Indépendamment des acceptions, le tableau 6 qui suit dresse la fréquence des lemmes (qui peuvent comprendre plusieurs mots formes; pluriel et forme féminine) des correspondants de business classés aussi en fonction de la technique de traduction directe utilisée. 
Tableau 6. - Correspondants par procédés directs.

\begin{tabular}{l|ll}
\multicolumn{1}{l|}{ Correspondant } & TC & RS \\
\hline entreprise & 19 & \\
affaire & 11 & \\
activité & 6 & \\
commercial & 6 & \\
économique & 3 & \\
commerce & 2 & \\
secteur & 2 & \\
cantine & 1 & \\
compagnie & 1 & \\
traite & 1 & 2 \\
business & & 2 \\
étude & & 1 \\
initiative & & 1 \\
activité & & 1 \\
capital & & 1 \\
entrepreneur & & 1 \\
jour & & 12 \\
ouvrable & & \\
positif & & \\
Total & 52 & \\
\end{tabular}

Même si le correspondant le plus fréquent (entreprise) peut être associé assez facilement à l'acception 3a, c'est déjà moins évident pour le deuxième correspondant le plus fréquent ( affaire) qui peut être associé aussi bien à l'acception 2a, 3a ou 3b. Malgré le caractère vague des liens qui peuvent s'établir entre les acceptions de business et les correspondants utilisés pour les traduire, les données du tableau 6 permettent aussi de repérer les anomalies de traduction ou les traductions problématiques qui attestent de la présence d'erreurs de traduction, comme c'est le cas du lexème jour, de ruptures sémantiques ou même de collocations.

Par exemple, dans les RS, les occurrences de business en français illustrent des emprunts qui s'expliquent par l'utilisation de deux noms propres complexes non traduits. D'autres traductions comme capital, positif ou ouvrable, sont révélatrices de l'existence de collocations ou mots composés compositionnels (dont le sens est égal à la somme de leurs parties) dont le statut d'unité lexicale n'est pas clairement établi ou qui ne sont pas décrites comme telles dans les dictionnaires bilingues, comme c'est le cas de big business = grand capital, $i t$ 's good business $=$ c'est positif et business day $=$ jour ouvrable.

En revanche, deux RS mettant en cause les traductions entrepreneurs et activité induisent plutôt un glissement conceptuel qui transforme l'acception du concept évoqué. Le tableau 7 qui suit fournit les énoncés dans lesquels figurent ces deux ruptures sémantiques (soulignées) et met en évidence l'exactitude de la traduction malgré le glissement conceptuel (et une nouvelle réfutation bien concrète de l'objection préjudicielle de la traduction, c'est-à-dire de son impossibilité théorique).

L'étude des ruptures sémantiques est ainsi révélatrice de phénomènes récurrents comme les collocations ou les glissements de sens qui témoignent des difficultés particulières de la traduction et des procédés de traduction non conventionnels qui respectent le principe 
de la traduction miroir de type mot à mot sans pour autant miser sur une traduction littérale.

Tableau 7. - Deux exemples de correspondant par solution directe.

Segment source
A fourth key measure that I would like to highlight
is the financial support that the act proposes
providing to the Canadian Youth Business
Foundation to encourage Canada's young
entrepreneurs.
Provided that they are based upon the different
levels of productivity according to region, these
differentiations would not lead to any business
relocations from one region or from another.

Segment cible

Quatrièmement, je souligne le soutien financier que ce projet de loi propose de fournir à la Fondation canadienne des jeunes entrepreneurs, afin d'encourager les jeunes entrepreneurs du Canada. A condition qu'elles soient basées sur les différents niveaux de productivité selon les régions, ces différenciations n'entraîneraient aucun déplacement d'activité d'une région ou d'une autre.

Ces deux exemples montrent que le sens qui se dégage des énoncés peut très bien s'exprimer dans une autre langue avec des moyens linguistiques (des acceptions) différents. Le premier exemple de RS de ce type est celui dans lequel la traduction réduit en quelque sorte l'acception $3 b$ (" all profit-making entities in society ») de business aux personnes qui animent ce secteur d'activité, un concept qui n'est pas lexicalisé avec business, mais avec un autre terme (entrepreneur), lequel est même un dérivé de l'acception 3a. Dans le deuxième cas, la traduction fait glisser l'acception 3a (« a specific business concern ») vers l'acception 2a qui renvoie à des activités (commerciales et économiques). Ce dernier adjectif, économique, se retrouve même parmi les traductions conventionnelles de business.

\subsection{Les procédés en fonction des patrons syntaxiques}

Une corrélation intéressante pour l'apprentissage de la traduction et pour la description des phénomènes récurrents dans les techniques de traduction est celle que l'on peut établir entre la construction syntaxique dans laquelle on trouve business et la technique de traduction utilisée dans le segment cible correspondant. Le tableau 8 qui suit décrit la répartition des deux principales techniques de traduction (directe ou indirecte) en fonction des patrons syntaxiques.

Tableau 8. - Modalités de traduction en fonction du patron syntaxique.

\begin{tabular}{l|ll} 
Patron syntaxique & $\begin{array}{l}\text { Solutions } \\
\text { directes }\end{array}$ & $\begin{array}{l}\text { Solutions } \\
\text { indirectes }\end{array}$ \\
\hline UP sources (16) & $9(56,3 \%)$ & $7(43,7 \%)$ \\
Modifieur (27) & $18(66,7 \%)$ & $9(33,3 \%)$ \\
Modifieur complexe (20) & $14(70 \%)$ & $6(30 \%)$ \\
Noyau (15) & $12(80 \%)$ & $3(20 \%)$ \\
Emploi unitaire (13) & $11(84,6 \%)$ & $2(15,4 \%)$ \\
Total (91) & $64(70,3 \%)$ & $27(29,7 \%)$
\end{tabular}

On y indique le nombre d'occurrences de chaque patron (première colonne) qui a été traduit directement (deuxième colonne) ou indirectement (troisième colonne). Le pourcentage indique le rapport entre le procédé de traduction utilisé et l'ensemble des 
occurrences du patron syntaxique. Le pourcentage est arrondi à l'entier supérieur ou inférieur le plus proche.

Les données du tableau 8 font ressortir le fait que $70 \%$ de toutes les occurrences de business sont traduites directement par correspondance, et que $85 \%$ des emplois unitaires sont traduits par une technique de traduction directe (par correspondance). Aussi, on constate que seulement $56,3 \%$ des occurrences de business dans une UP sont traduites par une solution directe, la proportion la plus faible des patrons syntaxiques traduits directement. On constate aussi une progression régulière et intéressante du pourcentage de solutions de traduction directes, qui passe de $56 \%$ à $85 \%$, des données en corrélation avec le caractère discret ou autonome du concept de business dans ses emplois syntaxiques. Ce fait de traduction concorde avec la conception selon laquelle les relations comme les collocations et les autres faits grammaticaux des langues ne sont pas traduisibles directement parce qu'elles sont inédites dans les autres langues. De même, on constate que plus les éléments conceptuels d'une langue sont en relation privilégiée (collocation) ou inédite (unités phraséologiques) avec d'autres concepts, plus ces éléments nécessitent une solution de traduction indirecte qui ne se transpose pas directement dans l'autre langue.

\subsection{Répartition croisée des solutions de traduction}

Nous présentons ci-après une analyse originale de la répartition des procédés de traduction utilisés pour chaque acception en fonction de leur patron syntaxique. À notre connaissance, ce type d'analyse n'a jamais été mené dans l'évaluation des procédés de traduction. Il s'agit plus précisément d'examiner la répartition des solutions de traduction en fonction des acceptions et des patrons syntaxiques pour déterminer dans quelle mesure les procédés de traduction sont liés à des valeurs particulières de ces deux variables. Les tableaux $9 a$ et $9 b$ qui suivent présentent les occurrences qui ont été traduites directement pour chaque acception et pour chaque patron syntaxique, d'abord en nombre dans le tableau $9 \mathrm{a}$, puis en proportion du nombre total d'occurrences de chaque patron pour chaque acception dans le tableau $9 \mathrm{~b}$.

Tableau 9a. - Occurrences traduites directement.

\begin{tabular}{l|cccccc}
\multicolumn{1}{c}{ Acceptions $\rightarrow$} & $1 a$ & $1 b$ & $2 a$ & $3 a$ & $3 b$ & $3 c$ \\
Patrons $\downarrow$ & $(0)$ & $(6)$ & $(22)$ & $(24)$ & $(9)$ & $(2)$ \\
\hline UP sources (9) & - & $2 / 4$ & $4 / 8$ & $3 / 3$ & - & $0 / 1$ \\
Modifieur (18) & $0 / 1$ & - & $11 / 14$ & $5 / 5$ & $2 / 6$ & $0 / 1$ \\
Modif. complexe (14) & - & - & $0 / 3$ & $8 / 10$ & $6 / 7$ & - \\
Noyau (12) & - & $5 / 6$ & $3 / 4$ & $3 / 4$ & - & $1 / 1$ \\
Emploi unitaire (11) & - & - & $4 / 5$ & $5 / 5$ & $1 / 2$ & $1 / 1$ \\
Total (64) & $0 / 1$ & $7 / 10$ & $22 / 34$ & $24 / 27$ & $9 / 15$ & $2 / 4$
\end{tabular}


Tableau 9b. - Occurrences traduites directement (\%).

\begin{tabular}{l|cccccc}
\multicolumn{1}{c}{$\begin{array}{c}\text { Acceptions } \rightarrow \\
\text { Patrons } \downarrow\end{array}$} & $1 a$ & $1 b$ & $2 a$ & $3 a$ & $3 b$ & $3 c$ \\
\hline UP sources (9) & $(0)$ & $(6)$ & $(22)$ & $(24)$ & $(9)$ & $(2)$ \\
Modifieur (18) & - & 50 & 50 & 100 & - & 0 \\
Modif. complexe (14) & - & - & 79 & 100 & 33 & 0 \\
Noyau (12) & - & - & 0 & 80 & 86 & - \\
Emploi unitaire (11) & - & - & 83 & 75 & - & 100 \\
Total (64) & 0 & 70 & 65 & 89 & 60 & 50
\end{tabular}

Les procédés de traduction directs et indirects sont mutuellement exclusifs et entièrement discrets : aucune solution de traduction n'est à la fois directe et indirecte et chacune des solutions de traduction est directe ou indirecte. Le caractère discret des solutions de traduction fait en sorte que les données des tableaux $9 a$ et $9 b$ sur les traductions directes renseignent aussi sur les traductions indirectes. À titre d'exemple, la proportion de traductions directes la plus faible ( $33 \%$ ) dans le tableau $9 \mathrm{~b}$ qui s'applique aux acceptions $3 \mathrm{~b}$ utilisées comme modifieur correspond de façon complémentaire à la proportion de traductions indirectes la plus élevée (67\%) qui s'applique aux mêmes acceptions. Nous revenons ci-dessous sur ces données complémentaires.

Le tableau $9 \mathrm{~b}$ permet aussi de déterminer rapidement pour quelles acceptions et pour quels patrons syntaxiques on retrouve la plus grande proportion de traductions directes (par correspondance conventionnelle et par rupture sémantique) ou de traductions indirectes (par rupture formelle, par rupture formelle et sémantique, ou par tradaptation). On peut constater que les pourcentages de solutions de traduction directes sont assez élevés en général, et plus spécialement pour l'acception 3a comme nous avons déjà pu le constater à la section 5.3. De même, on constate aussi que l'acception $3 b$ dans le patron syntaxique d'une modification complexe est celle qui se traduit le plus fortement au moyen de solutions de traduction directes (dans une proportion de $86 \%$ ). Les six correspondants (sur 7 occurrences) de cette acception et de ce patron syntaxique sont: affaires (2), business (calque, dans un nom propre), économique (2) et entrepreneurs. Si on met en parallèle ces différentes traductions avec le sens de cette acception, il semble bien que le concept des affaires (au pluriel) soit celui qui convienne le plus directement à cette acception. Cela dit, cela n'empêche pas non plus de constater que les deux concepts, business $3 \mathrm{~b}$ et affaires, renvoient à des notions bien différentes, soit à un ensemble d'opérations économiques en français, et à un ensemble d'agents économiques ou de sociétés en anglais. Ces autres traductions avec affaires montrent que des unités lexicales dont l'acception diffère de l'acception du terme auquel elles correspondent en langue source peuvent néanmoins rendre le même sens.

Pour ce qui est de la répartition des solutions de traduction indirectes, on trouve aussi que lorsque l'acception $3 b$, qui désigne le secteur privé, est employée comme modifieur, $67 \%$ des occurrences sont traduites indirectement (selon différents types de ruptures formelles et sémantiques). Une partie des explications dans ce cas-ci peut être attribuable au fait que la langue française doit recourir à des expressions comme monde des affaires ou milieu des affaires, gens d'affaires ou secteur privé pour désigner l'ensemble des entités et agents économiques qui mènent des activités à but lucratif dans une économie déterminée et que ces syntagmes nominaux peuvent être difficilement utilisés comme élément modifieur dans un syntagme nominal ayant déjà un noyau syntaxique. Il se peut 
que la plus grande facilité de traduction de la fonction de modifieur complexe pour l'acception $3 \mathrm{~b}$ vienne du fait que ce type de complément se rende plus couramment avec un complément comme « des affaires » ou une apposition.

\section{Analyse des résultats}

traduction directes avec le type de construction syntaxique dans laquelle le concept de business apparaît : son niveau le plus bas étant atteint avec les UP et son niveau le plus haut étant atteint avec ses emplois unitaires. Ces données mettent en évidence la difficulté croissante de la traduction à mesure que les concepts à traduire s'emploient de manière autonomes (emplois unitaires ou en tant qu'élément noyau), qu'ils entretiennent des liens étroits et qu'ils dépendent de plus en plus d'un autre concept, lorsqu'ils sont employés comme modifieur ou comme modifieur complexe. En fin de compte, ces données corroborent l'intuition selon laquelle les UP sont les éléments linguistiques dont le sens est le plus difficile à traduire d'une langue à une autre en inscrivant les UP à l'extrémité d'un continuum de combinatoires libres et sémantiquement régulières qui s'opposent à des combinatoires irrégulières et opaques.

ce qui concerne la vue d'ensemble des procédés directs de traduction, on a constaté que les ruptures sémantiques jouent un rôle essentiel en traduction puisqu'elles représentent la manifestation concrète de la possibilité d'exprimer des sens similaires au moyen d'acceptions ou de désignations différentes. difficile d'évaluer le rôle qualitatif des ruptures dans les solutions de traduction de business parce qu'on ne dispose pas de données quantitatives comparables sur la traduction d'autres concepts, leur nombre relativement élevé montre bien à la fois le rôle limité des dictionnaires bilingues qui ne peuvent recenser la totalité des traductions d'un concept, et la reproductibilité des mécanismes (hyperonymies, hyponymies, métonymies et glissements sémantiques) qui sont à l'œuvre dans les ruptures sémantiques et dans la traduction de business sur le plan uniquement conceptuel (sans prise en compte des annotations syntaxiques). Enfin, toujours sur le plan conceptuel, on a vu que près de $30 \%$ des solutions de traduction de business sont des traductions indirectes. Pour bien mesurer la particularité de business par rapport à d'autres concepts, il faudra étudier d'autres concepts spécialisés pour disposer d'analyses comparables.

Les données croisées des tableaux 9 ont montré que l'acception $3 b$ dans le patron syntaxique de modifieur complexe est la traduction la plus facile de l'ensemble des acceptions avec un taux de traduction directe de $86 \%$. De même, l'acception $3 \mathrm{~b}$ dans le 
patron syntaxique de modifieur semble être la traduction la plus difficile de business en français du fait que cette langue ne possède pas d'unité lexicale dont le générique est comparable à celui de l'acception $3 \mathrm{~b}$ de business («l'ensemble des entreprises d'une économie »). La langue française dans ce cas doit souvent procéder par omission, par étoffement ou par l'emploi d'un correspondant qui témoigne de l'emploi d'une acception dérivée qui permet néanmoins d'exprimer un sens similaire.

\section{Conclusion et perspectives ouvertes}

Les données compilées sur les occurrences bilingues du concept de business en anglais traduites en français ont permis de caractériser les normes de traduction du concept spécialisé de business de l'anglais au français. Elles donnent un aperçu des généralisations sur des faits de langue et de traduction qui pourront être validées au moyen d'une analyse quantitative subséquente.

La méthode inédite qui a été déployée ici offre une nouvelle approche pour découvrir et compiler des normes de la traduction pragmatique réellement utilisées au moyen des méthodes de la linguistique de corpus et de la traductologie fondée sur des corpus bilingues. Les grandes catégories d'annotations et leurs sous-types ont permis de rendre compte des paramètres sémantiques et formels (constructions syntaxiques) qui caractérisent les normes de traduction des occurrences de business réparties en deux grands groupes: les traductions directes par correspondance (conventionnelles ou non) et les traductions indirectes qui procèdent d'une rupture formelle ou formelle et sémantique avec la formulation en langue source.

Les résultats obtenus avec la présente étude pilote montrent de quelle façon il serait possible d'exploiter de manière quantitative les très nombreux corpus parallèles auxquels les traductologues ont désormais accès en vue de décrire les procédés et les normes de traduction des concepts spécialisés. Ces normes seront indispensables à la compréhension des particularités de la traduction des concepts nominaux spécialisés en langue de spécialité et conséquemment à l'apprentissage et à l'évaluation de la traduction spécialisée professionnelle.

Le présent article ouvre la voie à un traitement quantitatif des traductions du concept business anglais-français à l'aide d'annotations automatisées dans des corpus parallèles. Pour les annotations sémantiques, les possibilités d'automatisation sont à envisager dans le cadre de la désambiguïsation des mots et de l'attribution d'acceptions à des occurrences d'unités lexicales. Le traitement quantitatif des annotations syntaxiques et traductologiques est envisageable grâce aux analyseurs syntaxiques (parseurs) automatiques qui permettent de représenter la structure syntaxique d'énoncés en anglais et en français, et, jusqu'à un certain point, aux outils d'alignement automatiques qui permettraient de déterminer le correspondant ou l'occurrence vide qui est associée en langue cible à la traduction de business. On trouve une liste importante de ces outils (la plupart en accès libre) dans la rubrique de logiciels de Wikipedia (2018), une technologie qui a fait ses preuves pour l'alignement des segments des corpus parallèles (bitextes), mais qui reste à valider pour ce qui est de l'alignement des mots à l'intérieur des segments. En effet, ces outils ne semblent pas être en mesure de réaliser adéquatement l'alignement de mots complexes multilexémiques en même temps qu'ils discriminent ces mots et expressions complexes des mots simples. Une stratégie envisageable, inspirée de la méthode que nous avons décrite ici, consiste à mener l'analyse en deux temps en 
ciblant d'abord les unités phraséologiques puis en procédant ensuite à l'analyse des mots simples résiduels.

\section{BIBLIOGRAPHIE}

BAKKeR Matthijs, Koster Cees \& VAn Leuven-ZwART Kitty (2011), « Shifts », M. Baker \& G. Saldanha (dir.), Routledge Encyclopedia of Translation Studies, ( $2^{\mathrm{e}}$ éd.), Londres et New York : Routledge, 269-274.

BeEby Alison, Fernández Mónica, Fox Olivia, Kozlova Inna, Neunzig Wilhelm [...] HuRTado AlbiR Amparo (2005), « Investigating Translation Competence: Conceptual and Methodological Issues », Meta : journal des traducteurs / Meta: Translators' Journal, 50(2), 609-619, <http://id.erudit.org/ iderudit/011004ar>.

DESLILE Jean \& Fiola Marco A. (2013), La traduction raisonnée. Manuel d'initiation à la traduction professionnelle de l'anglais vers le français ( $3^{\mathrm{e}}$ éd.), Presses de l'Université d'Ottawa.

Dictionnaire électronique des synonymes (DES) (2008-2018), Centre de recherche inter-langues sur la signification en contexte (Crisco), Université de Caen Normandie, en ligne sur < www.crisco.unicaen.fr/des/> (16 avril 2018).

FRANCO AIXELÀ Javier (2015), « Anisomorfismos y traducción », Enciclopedia Abierta de Estudios de Traducción e Interpretación (AIETI), <www.aieti.eu/Enciclopedia/ANI-iconoses/index.html> (16 avril 2018).

GLEDHILL Christopher (2011), « A Lexicogrammar Approach to Checking Quality: Looking at One or Two Cases of Comparative Translation », I. Depraetere (dir.), Perspectives on Translation Quality, Mouton de Gruyter, 71-98, <www.eila.univ-paris-diderot.fr/_media/user/christopher_gledhill/ lexicogrammar_approach_to_translation_draft_gledhill.pdf> (16 avril 2018).

Gries Stefan T. (2008), «Phraseology and Linguistic Theory: A Brief Survey », S. Granger \& F. Meunier (dir.), Phraseology: An Interdisciplinary Perspective, Amsterdam et Philadelphie : John Benjamins, 3-25.

HANKS Patrick (2013), Lexical Analysis: Norms and Exploitations, MIT Press.

HUMBLEY John (2006), « La néologie : interface entre ancien et nouveau », R. Greenstein (dir.), Langues et cultures : une histoire d'interface, Publications de la Sorbonne, 91-103, <halshs-00168574> (16 avril 2018).

OFFICE QUÉBÉCOIS DE LA LANGUE FRANÇAISE (2018), « Les modificateurs », en ligne sur Banque de dépannage linguistique : <http://bdl.oqlf.gouv.qc.ca/bdl/gabarit_bdl.asp?T1=les + modificateurs\&T3.x=0\&T3.y=0\&id=4288>.

POIRIER Éric (2015), « The Interpretation of 'Business' in Specialized Expressions and Compound Terms for Translation Purposes », Intralinea Online Translation Journal, 17, <www.intralinea.org/ specials/article/the_interpretation_of_business_in specialized_expressions> (16 avril 2018). 
POIRIER Éric (2016a), « Exploring Theoretical Functions of Corpus Data in Teaching Translation », Cadernos de Tradução, 36(1), <https://periodicos.ufsc.br/index.php/traducao/article/ view/2175-7968.2016v36nesp1p177> (16 avril 2018).

POIRIER Éric (2016b), « Meaning-Based Textual Word Alignment Heuristic », R. Chbeir, R. Agrawal \& I. Biskri (dir.), Proceedings of the 8th International Conference on Management of Digital EcoSystems (MEDES'16) Conference, Association for Computer Machinery (ACM), 208-214.

SINCLAIR John (1991), Corpus, Concordance, Collocation, Oxford : Oxford University Press.

TOURY Gideon (2012), Descriptive Translation Studies - and beyond (Revised edition), Amsterdam/ Philadelphie : John Benjamins.

TRADOOIT CONCORDANCIER BILINGUE (2017), Rimouski : Okidoo, en ligne sur <www.tradooit.com> (16 avril 2018).

TUTIN Agnès \& GRosSmANN Francis (2002), « Collocations régulières et irrégulières : esquisse de typologie du phénomène collocatif », Revue française de linguistique appliquée, 7(1), 7-25.

WeCKSTEen-Quinio Corinne, MARIAule Mickaël \& LefebVRe-Scodeller Cindy (2015), La traduction anglais-français. Manuel de traductologie pratique, Louvain-la-Neuve : De Boeck.

WIKIPEDIA (2018), Bitext word alignment, en ligne sur <https://en.wikipedia.org/wiki/ Bitext_word_alignment> (16 avril 2018).

\section{NOTES}

1. L'échantillon de 100 occurrences au départ a dû être réduit à 91 en cours d'analyse de manière à supprimer les doublons dans le corpus (mêmes occurrences source et cible) ainsi que des occurrences des acceptions de business appartenant à la langue générale.

2. Cela n'empêche pas qu'il puisse se glisser des erreurs dans ces traductions que la compilation des normes de traduction pourra justement mettre en évidence du fait de l'anomalie qu'elles incarnent par rapport aux régularités dégagées.

3. «In sum, a phraseologism is defined as the co-occurrence of a form or a lemma of a lexical item and one or more additional linguistic elements of various kinds which functions as one semantic unit in a clause or sentence and whose frequency of cooccurrence is larger than expected on the basis of chance. "

4. Comme nous avons vu, l'échantillon a ensuite été réduit à 91 par suite de la suppression des doublons et des occurrences de la langue générale.

5. À cette étape-ci, les UP du texte source ont déjà été repérées à l'étape des annotations syntaxiques et le texte source ne devrait contenir que des unités lexicales simples. Le traitement des UP du texte cible est effectué manuellement lorsque nécessaire par l'alignement de deux unités lexicales simples du texte cible à une unité lexicale simple du texte source.

\section{RÉSUMÉS}

Nous proposons une méthode de découverte et de compilation des normes de traduction des concepts spécialisés employés dans des termes simples et complexes attestés dans un corpus 
parallèle bilingue. Les normes de traduction mises au jour par cette méthode ont la particularité d'être fondées sur l'usage et prennent appui sur des solutions de traduction éprouvées. Celles-ci sont essentielles à l'enseignement des compétences en traduction spécialisée telles que proposées par le groupe PACTE et généralement acceptées en traductologie. Notre méthode consiste à analyser la traduction des occurrences spécialisées de business en économie et en finance réunies dans un corpus bilingue constitué d'un échantillon d'occurrences aléatoires obtenues au moyen d'un concordancier en ligne. L'analyse repose sur trois catégories d'annotations et leurs corrélations: l'acception de business, la fonction de business dans le syntagme nominal et les modalités de traduction de business. Cette méthode d'analyse peut être facilement étendue à l'ensemble des concepts spécialisés de nature nominale qui sont des unités distinctives des textes de spécialité.

We propose a method for the discovery and compilation of translation standards of specialized concepts used in simple and complex terms attested in a parallel bilingual corpus. The standards of translation developed by this method have the characteristic of being based on usage and are based on proven translation solutions. These are essential to the teaching of specialized translation skills, as proposed by the PACTE group and generally accepted in translation studies. Our method consists in analysing the translation of specialized tokens of business in economics and finance collected in a bilingual corpus made up of a sample of random occurrences generated with an online concordancer. The analysis is based on three categories of annotations and their correlations: senses of business, functions of business in the noun phrase and translation modes of business. This method of analysis can be easily extended to all specialized concepts of a nominal nature, which are distinctive units of specialized texts.

INDEX

Keywords : translation norms, specialized translation, bilingual parallel corpora, business, textual correlations

Mots-clés : normes de traduction, traduction spécialisée, corpus bilingues parallèles, business, corrélations textuelles

\section{AUTEUR}

\section{ÉRIC POIRIER}

Université du Québec à Trois-Rivières 